\title{
Utilidad de la ecocardiografía transtorácica focalizada en emergencias hemodinámicas intraoperatorias
}

\author{
Usefulness of Focalized transthoracic ecocardiography \\ in intraoperative hemodynamic emergencys
}

María Carolina Cabreraํ, Andrea Straub

\begin{abstract}
Introduction: Echocardiography represents one of the most important advances in the monitoring of critical patients. Initially available only in cardiovascular surgery, currently, there is transesophageal echocardiography (TEE) and transthoracic echocardiography (ETT) in non-cardiac surgery, for anesthesiologists. The advantages of ETT is a non-invasive tool, of lower cost than the transesophageal transducer and therefore more feasible to be overcrowded and available in the pavilion. Objective: To evaluate the usefulness of TTE in patients with hemodynamic compromise during non-cardiac surgery. Material and Methods: In a prospective manner between April 2016 and September 2018, patients were studied who during their intraoperative period presented a compromise of their hemodynamic state, defined as an average blood pressure under $55 \mathrm{~mm} \mathrm{Hg}$, for more than 3 minutes and without response to the usual therapy based on vasopressors and volume. Each of these patients had a prospective protocol for focused ETT looking for the cause of this disorder, by a duly trained operator. Results: 124 patients, with an average age of 67 years (range 42 to 93 years) were evaluated. In all cases, at least one echocardiographic window was obtained that allowed a diagnosis to be made and/or to guide the therapy. The main causes of hemodynamic compromise were hypovolemia $(52 \%)$, poor left ventricular function (21\%) and other causes such as pericardial effusion, suspected pulmonary thromboembolism, pulmonary pathology and suspected myocardial ischemia. Discussion: The ETT could be a feasible tool to use in acute hemodynamic events, since it offers good quality windows that allow new decisions based on the diagnosis and also allows to guide the selected therapies. In addition, it has been shown to positively impact clinical
\end{abstract}

\section{Key words:}

Focused transthoracic echocardiography, intraoperative hemodynamic instability

Departamento de Anestesiología Universidad de Valparaíso. Valparaíso, Chile.

Fecha de recepción: 12 de agosto de 2019

Fecha de aceptación: 30 de noviembre de 2019

ORCID

https://orcid.org/0000-0002-5544-8000

Correspondencia:

María Carolina Cabrera

Email: maria.cabrera@uv.cl 
behaviors in the perioperative period. Conclusion: ETT is a non-invasive monitor, reasonably easy to learn to use; In addition to directly visualizing cardiac structures, it allows differential diagnoses of the causes of intraoperative hypotension. The therapies can also be decided according to the echocardiographic images and control how they generate changes in the cardiac cavities and in the hemodynamic state of the patient.

\section{RESUMEN}

Introducción: La ecocardiografía representa uno de los más importantes avances en la monitorización de pacientes críticos. Inicialmente disponible sólo en cirugía cardiovascular, en la actualidad, se cuenta con ecocardiografía transesofágica (ETE) y ecocardiografía transtorácica (ETT) en cirugía no cardíaca, para los anestesiólogos. Las ventajas del ETT están en ser una herramienta no invasiva, de menor costo que el transductor transesofágico y, por lo tanto, más factible de ser masificada y estar disponible en pabellón. Objetivo: Evaluar la utilidad de ETT en pacientes con compromiso hemodinámico durante cirugía no cardiaca. Material y Métodos: En forma prospectiva entre abril de 2016 y septiembre del 2018, se estudiaron enfermos que durante su intraoperatorio presentaron compromiso de su estado hemodinámico, definido como una presión arterial media bajo $55 \mathrm{~mm} \mathrm{Hg}$, por más de 3 minutos y sin respuesta a la terapia habitual basada en vasopresores y volumen. A cada uno de estos enfermos se le realizó un protocolo prospectivo de ETT focalizado buscando la causa de esta alteración, por un operador debidamente entrenado. Resultados: 124 pacientes, con edad promedio de 67 años (rango 42 a 93 años) fueron evaluados. En todos los casos se obtuvo al menos una ventana ecocardiográfica que permitió realizar un diagnóstico y/o guiar la terapia. Las principales causas de compromiso hemodinámico fueron hipovolemia (52\%), mala función del ventrículo izquierdo (21\%) y otras causas como derrame pericárdico, sospecha de tromboembolismo pulmonar, patología pulmonar y sospecha de isquemia miocárdica. Discusión: La ETT podría ser una herramienta factible de utilizar en eventos hemodinámicos agudos, ya que ofrece ventanas de buena calidad que permiten tomar decisiones nuevas basadas en el diagnóstico y, además, permite guiar las terapias seleccionadas. Además, ha mostrado impactar de forma positiva las conductas clínicas en el perioperatorio. Conclusión: La ETT es un monitor no invasivo, razonablemente fácil de aprender a utilizar que además de visualizar de manera directa las estructuras cardíacas, permite realizar diagnósticos diferenciales de las causas de hipotensión intraoperatoria. Además se puede decidir las terapias de acuerdo a las imágenes ecocardiográficas y controlar cómo éstas generan cambios en las cavidades cardíacas y en el estado hemodinámico del paciente.

\section{Palabras clave:}

Ecocardiografía transtorácica focalizada, inestabilidad hemodinámica intraoperatoria

\section{Introducción}

a ecografía in situ o en el punto de atención (PoCUS por su sigla en inglés) ha sido incorporada _ por los anestesiólogos para facilitar la punción de los accesos venosos centrales y la anestesia regional hace más de una década. La utilidad para el manejo de emergencias hemodinámicas había sido relegada a las especialidades de medicina de urgencias y cuidados críticos. Sin embargo, en el último tiempo, el PoCUS perioperatorio ha surgido para esclarecer dudas en patología sistémica, tales como patología cardíaca, patología pulmonar y estimación de presión intracraneana entre otras[1],[2]. 
La ecocardiografía representa uno de los avances más importantes en la monitorización de paciente crítico, tanto en el contexto perioperatorio como en $\mathrm{UCl}$, siendo un monitor no invasivo en tiempo real[3]. El uso de ecocardiografía se remonta a los años 80 por los anestesiólogos cardiovasculares con excelentes resultados y beneficios para el paciente. Debido a esto, en la actualidad su uso es de rutina[4]. Además, tanto la ecocardiografía transesofágica (ETE) como la ecocardiografía transtorácica (ETT), ya son utilizadas de manera rutinaria para la monitorización del estado hemodinámico tanto de manera electiva como aguda en cirugía no cardíaca[1],[5].

La ecocardiografía entrega un panorama fisiopatológico de los desajustes hemodinámicos, permitiendo ser interpretado para tomar decisiones terapéuticas más apropiadas[5],[6].

La ETT focalizada aplicada por anestesiólogos en el período perioperatorio es factible y podría alterar el manejo[4],[7]. Entre las ventajas de la ETT se encuentran que es una herramienta no invasiva, no se han reportado efectos adversos por su uso, tiene menor costo que la ETE, y que hay más factibilidad para su disponibilidad en pabellón. Ha tenido una introducción explosiva en los últimos años, teniendo habitualmente el tamaño de un computador portátil (el equipo va al paciente), y ha permitido que más colegas tengan acceso a la técnica[4],[8].

Al ser una herramienta no invasiva, permite su aprendizaje tanto en voluntarios vivos como en simuladores. Su entrenamiento de adquisición de imágenes de ecocardiografía transtorácica por medio de simulación ha mostrado ser no inferior al entrenamiento en voluntarios vivos; permite, además, aprender conceptos y procedimientos más complejos, y otorga la oportunidad de entrenar habilidades no técnicas como manejo de tareas, liderazgo, trabajo en equipo y toma de decisiones[9]-[12]. El enfrentamiento FoCUS (Focalized Cardiovascular UltraSound) propone el ultrasonido como una extensión del examen físico del paciente[13],[14]. Su utilidad en el período perioperatorio ha sido demostrada en momentos críticos y para toma de decisiones clínicas tiempo sensibles[15]. Las indicaciones más comunes en el perioperatorio incluyen signos o síntomas de insuficiencia cardiaca, e inestabilidad hemodinámica. Los objetivos de FoCUS incluyen evaluación estructural cardiaca sistólica biventricular, de la función valvular, pericárdica y del estado de volumen[13].

\section{Protocolos de ecocardiografía transtorácica}

La ETT focalizada se refiere a la aplicación de ultrasonido breve, orientada a objetivos, habitualmen- te realizada por el médico tratante y en el punto de atención o al lado de la cama del paciente. Tal intervención está diseñada para una evaluación rápida de la función y el estado cardiacos, especialmente cuando el tiempo apremia y la ecocardiografía formal no está disponible de forma oportuna[16],[17]. A lo largo de los años, se ha planteado múltiples protocolos para enfrentar de manera enfocada y ordenada el examen en diversos contextos clínicos. Todos comparten ciertas características, siendo orientados a un problema, intentan responder a preguntas dicotómicas, consumen poco tiempo, son repetibles (lo cual permite evaluar sin retraso el efecto de intervenciones realizadas) y no requieren el traslado del paciente. Su implementación ha sido facilitada por el progreso tecnológico con mejor calidad de imagen, alta portabilidad y equipos de menor costo[4],[18],[19].

Uno de los primeros protocolos y probablemente el más utilizado y probado es el FATE (Focused Assessed Transthoracic Echo)[20]. Es un protocolo simple para interpretar hallazgos ecocardiográficos en un contexto clínico que permite descartar patología obvia. Pretende el uso de la interpretación cualitativa y el reconocimiento de patrones en ETT básica, así como el uso de mediciones Doppler en manos entrenadas. Sus indicaciones incluyen la presencia de soplos sistólicos, sospecha de patología valvular, inestabilidad hemodinámica, evaluación de la función ventricular, disnea no explicada, hipoxemia y limitación de la capacidad funcional[18]. Ayuda a resolver conflictos circulatorios en cinco pasos: 1. Buscar patología obvia; 2. Evaluar grosor de pared y dimensiones de cavidades cardiacas; 3. Evaluar función biventricular 4. Visualizar la pleura a ambos lados y 5 . Relacionar la información con el contexto clínico[19].

\section{Objetivo}

El objetivo principal de este estudio fue evaluar la utilidad de ETT, específicamente el protocolo FATE, en pacientes con compromiso hemodinámico durante cirugía no cardiaca. Se plantearon como objetivos secundarios describir los hallazgos ecocardiográficos de pacientes con desbalance hemodinámico intraoperatorio y determinar los cambios de conducta del anestesiólogo tratante según los hallazgos.

\section{Materiales y Métodos}

El estudio realizado es observacional, descriptivo y prospectivo. Los datos se obtuvieron entre abril del 
año 2016 y septiembre del año 2018 en el Hospital Clínico de la Fuerza Aérea de Chile. Se evaluó a adultos con compromiso de su estado hemodinámico, definido como presión arterial media menor a $55 \mathrm{~mm}$ de $\mathrm{Hg}$ por más de 3 minutos, y sin respuesta a terapia habitual (vasopresores y volumen a discreción del anestesiólogo tratante). Se aplicó un protocolo prospectivo de ETT focalizado[18] por un operador debidamente entrenado. Se utilizó una sonda ecográfica de 1-5 MHz, y se usó el protocolo FATE (Figura 1).

\section{Ventanas ecográficas}

Cuatro cámaras subcostal: el transductor se posiciona cerca de la línea media y justo bajo la curvatura torácica. El marcador de orientación de la sonda apunta a la izquierda del paciente, y el plano se alinea con el eje del corazón.

Cuatro cámaras apical: el transductor se posiciona sobre el ápex cardiaco. El marcador de orientación de la sonda apunta a la izquierda del paciente y el plano ecográfico se alinea con el eje cardiaco.

Eje largo y eje corto paraesternales: el transductor se posiciona paraesternal izquierdo, en una línea entre el ápex del corazón y el medio de la clavícula dere- cha del paciente. El eje largo aparecerá al apuntar el marcador de orientación de la sonda hacia el hombro derecho del paciente y el eje corto al rotar la sonda $90^{\circ}$ en sentido horario.

Escaneo pleural: el transductor se posiciona en la línea axilar posterior. La identificación del diafragma facilita la separación del contenido torácico del abdominal, y se puede observar potencial derrame pleural o atelectasias hacia cefálico.

Otras ventanas de interés: vena cava subcostal, dos cámaras apical, eje largo apical, cinco cámaras apical, eje corto paraesternal en plano mitral y a nivel de válvula aórtica[18].

\section{Resultados}

Se evaluó a un total de 124 pacientes adultos. La edad promedio fue de 67 años (rango 42 a 93 años). La obtención de ventanas ecocardiográficas que permitieran realizar un diagnóstico y/o guiar la terapia fue de al menos una en $100 \%$ de los casos, al menos dos en $78 \%$, y al menos tres en $42 \%$ de los pacientes.

En la Tabla 1 se resumen las causas interpretadas

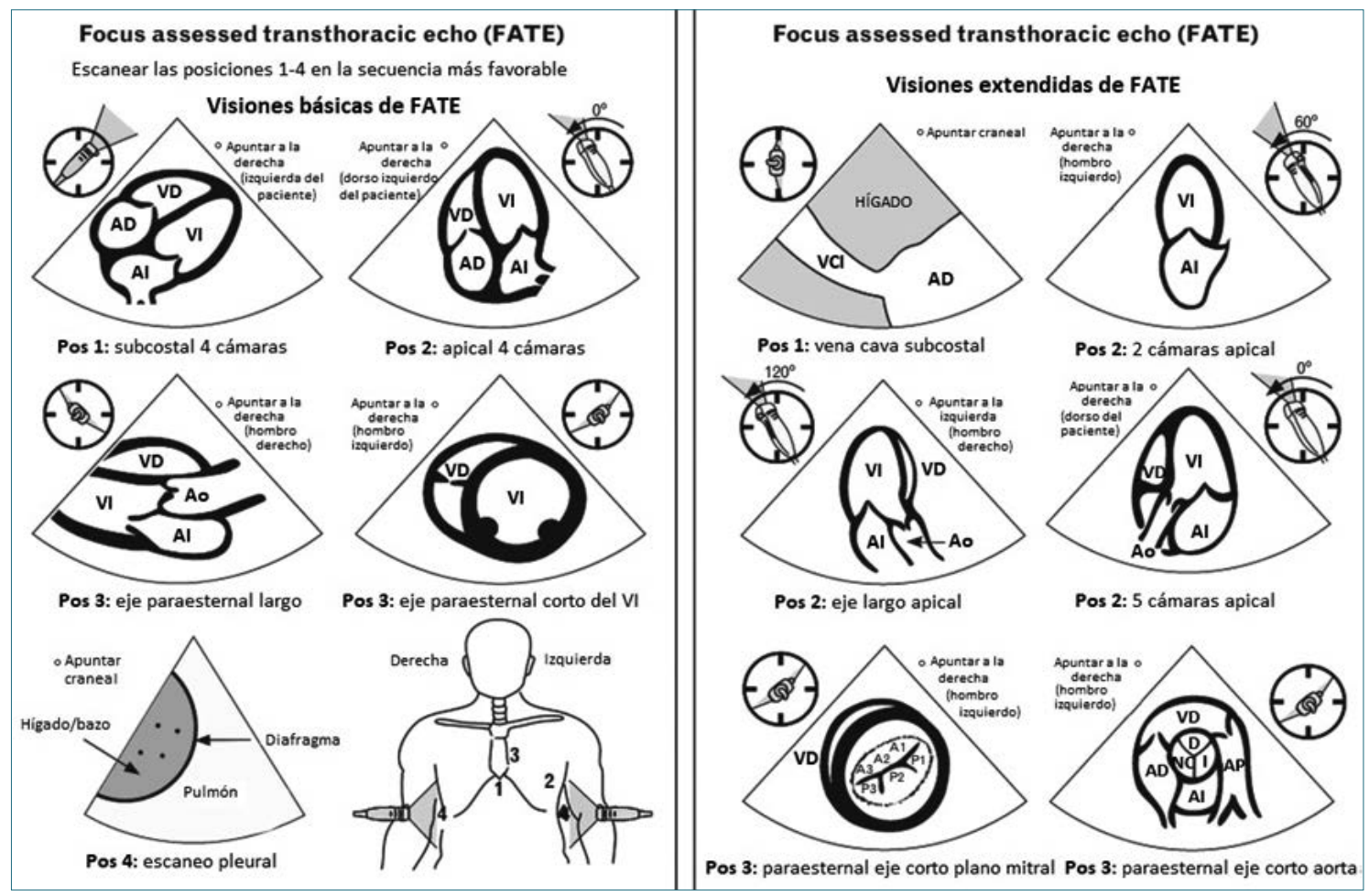

Figura 1. Protocolo FATE, visiones básicas y exendidas. Ao: aorta; VCl: vena cava inferior; Al: aurícula izquierda; VI: ventrículo izquierdo; AP: arteria pulmonar; AD: aurícula derecha; VD: ventrículo derecho. 
Tabla 1. Causas de desbalance hemodinámico

\begin{tabular}{ll}
\hline Hipovolemia & $52 \%$ \\
$\begin{array}{l}\text { Mala función ventricular } \\
\text { izquierda }\end{array}$ & $21 \%$ \\
Otras causas* & $27 \%$ \\
\hline
\end{tabular}

*Otras causas: derrame pericárdico, sospecha de tromboembolismo pulmonar, patología pulmonar, sospecha de isquemia miocárdica

de desbalance hemodinámico.

Con respecto a la conducta adoptada, hubo un cambio en la forma de administración de fluidos (bolo/restrictivo) en 35\%, inicio de monitorización invasiva (instalación de línea arterial) en $22 \%$, cambio en el plan postoperatorio en el $18 \%$ de los casos, inicio de drogas vasoactivas en un $16 \%$, cambio en la cirugía en $11 \%$, e inicio de betabloqueo en el $6 \%$ de los pacientes.

\section{Discusión}

La ETT podría ser considerada como una herramienta diagnóstica ante eventos hemodinámicos agudos intraoperatorios, para guiar la toma de decisiones; y puede ser usada como un monitor no invasivo. Permite visualizar causas de cambios hemodinámicos, guiar la terapia y controlar cambios. La importancia de visualizar lo que pasa en las cavidades permite realizar diagnósticos certeros y reales. $Y$ frente a una hipotensión, no siempre su causa es la hipovolemia. $Y$ una de las enseñanzas más importantes que entrega la ecocardiografía es que una hipotensión no es sinónimo de hipovolemia. Hace algunos años, los trabajos de Canty et al. y Cowie et al., demostraron que la implementación de ETT focalizada por el anestesiólogo puede generar un impacto perioperatorio positivo alterando el manejo y terapia de los enfermos[21]-[23]. Zimmerman y Coker realizaron una revisión de las técnicas básicas necesarias para su ejecución[13].

La aproximación PAUSE (Perioperative Anesthesiology UltraSonographic Evaluation) fue propuesto por Adler et al., en el año 2016. Este plantea realizar pausas en diferentes momentos del período perioperatorio y utilizar ecografía para evaluar al paciente, expandiendo la información disponible sólo con el examen físico o los signos vitales. Subdivide la evaluación en preoperatoria (estado de volumen, evaluación de función cardíaca, evaluación valvular en caso de soplos cardíacos), intraoperatoria (inestabilidad hemodinámica persistente) y postoperatoria (hipoten- sión). Además, propone la utilización de ultrasonido durante el paro cardiaco y para evaluación de patología pulmonar[24].

El uso de ultrasonido como una herramienta diagnóstica segura requiere competencias básicas en la adquisición e interpretación de imágenes. Numerosos estudios han demostrado que con entrenamiento básico en ultrasonido el médico promedio e incluso estudiantes de medicina pueden aprender a obtener imágenes básicas y reconocer patología significativa[24]-[26]. Recientemente se ha incorporado a algunos programas de formación el PoCUS perioperatorio “Evaluación sonográfica focalizada de riesgo perioperatorio que involucra ultrasonido gastroabdominal, hemodinámico y transtorácico" (denominado FORESIGHT por su sigla en inglés), y se ha sugerido que tendría un impacto perioperatorio positivo[2],[27]. En un curso que evaluó la habilidad de anestesiólogos, urgenciólogos e intensivistas para identificar anormalidades mayores utilizando visiones limitadas de ETT; se encontró concordancia de hallazgos en $90-99 \%$ tras la evaluación por cardiólogos expertos, siendo la discordancia la mayoría de las veces debido a falsos positivos[16].

La American Heart Association (AHA) y el American College of Cardiology (ACC) recomiendan el uso de ecocardiografía para la evaluación de desbalances hemodinámicos agudos, persistentes y que amenacen la vida, en los cuales la función ventricular y sus determinantes son inciertos, y que no hayan respondido a tratamiento (evidencia nivel I)[8].

La aplicación de ultrasonido al lado de la cama del paciente en el contexto perioperatorio ha mostrado alterar de forma positiva el curso general de pacientes[22]. El uso de PoCUS focalizado en el perioperatorio se sugiere como una herramienta clínica, y, ciertamente, no pretende reemplazar a evaluación ecocardiográfica formal por cardiólogos entrenados[24].

Las limitaciones de la ETT incluyen la naturaleza operador dependiente del examen y a veces la inhabilidad de obtención de ventanas ecocardiográficas. Además, puede haber casos en los cuales el campo quirúrgico pueda limitar la obtención de imágenes. Sin embargo, a veces, como en cirugía de cabeza y cuello, la obtención de imágenes podrá ser más factible con ETT que con ETE[24].

Globalmente, el concepto de PoCUS perioperatorio ha mostrado un crecimiento importante en los últimos años ${ }^{1}$. La posterior innovación e integración de esta herramienta tiene un enorme potencial para impactar en el cuidado perioperatorio proporcionando respuestas en tiempo real a muchos eventos agudos, 
minimizando las "adivinanzas" del manejo perioperatorio.

\section{Conclusión}

El uso de PoCUS perioperatorio ha tenido un aumento exponencial en los últimos años. Utilizar la ETT en el período perioperatorio podría tener un impacto positivo en el manejo de pacientes, siempre y cuando sea realizado por personal entrenado. El aprendizaje de protocolos de ultrasonido perioperatorio puede permitir un enfrentamiento ordenado y organizado ante eventos agudos hemodinámicos, para ayudar en la toma de decisiones con más información en diversos contextos clínicos. Con el uso de ecocardiografía hemos llegado a una muy importante conclusión y es que hipotensión no es sinónimo de hipovolemia.

\section{Referencias}

1. Khoche $S$, Ramsingh D, Maus $\mathrm{T}$. The Year in Perioperative Echocardiography: Selected Highlights From 2016. J Cardiothorac Vasc Anesth. 2017 Oct;31(5):1554-61. https://doi. org/10.1053/j.jvca.2017.04.049 PMID:28826850

2. Deshpande R, Ramsingh D. Perioperative point of care ultrasound in ambulatory anesthesia: thinking beyond nerve blocks. Curr Opin Anaesthesiol. 2017 Dec;30(6):663-9. https://doi.org/10.1097/ AC0.0000000000000529 PMID:29016368

3. Jozwiak M, Monnet $X$, Teboul JL. Monitoring: from cardiac output monitoring to echocardiography. Curr Opin Crit Care. 2015 Oct;21(5):395401. https://doi.org/10.1097/ MCC.0000000000000236 PMID:26348419

4. Cowie BS. Focused transthoracic echocardiography in the perioperative period. Anaesth Intensive Care. 2010 Sep;38(5):823-36. https://doi.org 10.1177/0310057X1003800505 PMID:20865866

5. Guarracino F, Bertini P. Perioperative haemodynamic management: is echocardiography the right tool? Curr Opin Crit Care. 2014 Aug;20(4):4317. https://doi.org/10.1097/

\section{0000000000000111 PMID:24979552}

6. Ng A, Swanevelder J. Perioperative echocardiography for non-cardiac surgery: what is its role in routine haemodynamic monitoring? Br J Anaesth. 2009 Jun;102(6):731-4. https:// doi.org/10.1093/bja/aep100 PMID:19451153

7. Alfirevic A. Con: Perioperative Transthoracic Echocardiography Should Not Be an Integral Part of the Anesthesiology Residency Core Curriculum. J Cardiothorac Vasc Anesth. 2015 Aug;29(4):1086-8. https://doi. org/10.1053/j.jvca.2015.04.012 PMID:26279225

8. Poth JM, Beck DR, Bartels K. Ultrasonography for haemodynamic monitoring. Best Pract Res Clin Anaesthesiol. 2014 Dec;28(4):337-51. https://doi. org/10.1016/j.bpa.2014.08.005 PMID:25480765

9. Nazarnia S, Subramaniam K. Role of Simulation in Perioperative Echocardiography Training. Seminars in Cardiothoracic and Vascular Anesthesia. SAGE Publications; 2016 Aug;21(1):81-94. http://dx.doi. rg/10.1177/1089253216655874

10. Magoon R, Sharma A, Ladha S, Kapoor PM, Hasija S. Simulationbased transthoracic echocardiography: "An anesthesiologist's perspective". Ann Card Anaesth. 2016 Jul-Sep;19(3):511-5. https://doi.org/10.4103/09719784.185544 PMID:27397457

11. Sharma $V$, Fletcher SN. A review of echocardiography in anaesthetic and peri-operative practice. Part 2: training and accreditation. Anaesthesia. 2014 Aug;69(8):919-27. https:// doi.org/10.1111/anae.12709 PMID:24801304

12. Edrich $T$, Seethala RR, Olenchock BA, Mizuguchi AK, Rivero JM, Beutler SS, et al. Providing initial transthoracic echocardiography training for anesthesiologists: simulator training is not inferior to live training. J Cardiothorac Vasc Anesth. 2014 Feb;28(1):49-53. https://doi. org/10.1053/j.jvca.2013.07.011 PMID:24183827

13. Zimmerman JM, Coker BJ. The Nuts and Bolts of Performing Focused Cardiovascular Ultrasound (FoCUS). Anesth Analg. 2017 Mar;124(3):75360. https://doi.org/10.1213/ ANE.0000000000001861 PMID:28207445

14. Conlin F, Roy Connelly N, Raghunathan K, Friderici J, Schwabauer A. Focused Transthoracic Cardiac Ultrasound: A Survey of Training Practices. J Cardiothorac Vasc Anesth. 2016 Jan;30(1):102-6. https://doi. org/10.1053/j.jvca.2015.05.111 PMID:26296825

15. Conlin F, Connelly NR, Eaton MP, Broderick PJ, Friderici J, 
Adler AC. Perioperative Use of Focused Transthoracic Cardiac Ultrasound: A Survey of Current Practice and Opinion. Anesth Analg. 2017 Dec;125(6):187882. https://doi.org/10.1213/ ANE.0000000000002089 PMID:28537977

16. Royse CF, Haji DL, Faris JG, Veltman MG, Kumar A, Royse AG. Evaluation of the Interpretative Skills of Participants of a Limited Transthoracic Echocardiography Training Course (H.A.R.T.scan® course). Anaesthesia and Intensive Care. SAGE Publications; 2012 May;40(3):498-504. http://dx.doi.g/10.1177/031005 7x1204000316

17. Barber RL, Fletcher SN. A review of echocardiography in anaesthetic and peri-operative practice. Part 1: impact and utility. Anaesthesia. 2014 Jul;69(7):764-76. https://doi. org/10.1111/anae.12663 PMID:24773366

18. Jørgensen MR, Juhl-Olsen $P$, Frederiksen CA, Sloth E. Transthoracic echocardiography in the perioperative setting. Curr Opin Anaesthesiol. 2016 Feb;29(1):4654. https://doi.org/10.1097/ AC0.0000000000000271 PMID:26658177

19. Holm JH, Frederiksen CA, Juhl-Olsen P, Sloth E. Perioperative use of focus assessed transthoracic echocardiography (FATE). Anesth Analg. 2012 Nov;115(5):1029-32. https://doi.org/10.1213/

ANE.0b013e31826dd867

PMID:23051882

20. Frederiksen $C A$, Juhl-Olsen $P$, Nielsen DG, Eika B, Sloth E. Limited intervention improves technical skill in focus assessed transthoracic echocardiography among novice examiners. BMC Med Educ. 2012 Aug;12(1):65. https://doi.org/10.1186/14726920-12-65 PMID:22863138

21. Cowie B. Three years' experience of focused cardiovascular ultrasound in the perioperative period. Anaesthesia. 2011 Apr;66(4):268-73. https://doi.org/10.1111/ j.1365-2044.2011.06622.x PMID:21401539

22. Cowie B. Focused transthoracic echocardiography predicts perioperative cardiovascular morbidity. J Cardiothorac Vasc Anesth. 2012 Dec;26(6):989-93. https://doi. org/10.1053/j.jvca.2012.06.031 PMID:22920841

23. Canty DJ, Royse CF, Kilpatrick $D$, Bowman L, Royse AG. The impact of focused transthoracic echocardiography in the pre-operative clinic. Anaesthesia. 2012 Jun;67(6):618-25. https://doi.org/10.1111/ j.1365-2044.2012.07074.x PMID:22352785

24. Adler AC, Greeley WJ, Conlin F, Feldman JM. Perioperative anesthesiology ultrasonographic evaluation (PAUSE): A guided approach to perioperative bedside ultrasound. J Cardiothorac Vasc Anesth. 2016 Apr;30(2):521-9. https://doi. org/10.1053/j.jvca.2015.11.015 PMID:27013122

25. Díaz-Gómez JL, Pérez-Protto $S$, Hargrave J, Builes A, Capdeville $M$, Festic $E$, et al. Impact of a focused transthoracic echocardiography training course for rescue applications among anesthesiology and critical care medicine practitioners: a prospective study. J Cardiothorac Vasc Anesth. 2015;29(3):576-81. https://doi. org/10.1053/j.jvca.2014.10.013 PMID:25622973

26. Tanzola RC, Walsh S, Hopman WM, Sydor D, Arellano R, Allard RV. Brief report: Focused transthoracic echocardiography training in a cohort of Canadian anesthesiology residents: a pilot study. Canadian Journal of Anesthesia/Journal canadien d'anesthésie. Springer Science and Business Media LLC; 2012 Oct 25;60(1):32-7. http://dx.doi. org/10.1007/s12630-012-98118

27. Ramsingh D, Rinehart J, Kain Z, Strom S, Canales C, Alexander $B$, et al. Impact assessment of perioperative point-of-care ultrasound training on anesthesiology residents. Anesthesiology. 2015 Sep;123(3):670-82. https://doi.org/10.1097/ ALN.0000000000000776 PMID:26181338 